\title{
Article \\ New Conservation Laws and Exact Cosmological Solutions in Brans-Dicke Cosmology with an Extra Scalar Field
}

\author{
Antonios Mitsopoulos ${ }^{1}$, Michael Tsamparlis ${ }^{1}$, Genly Leon ${ }^{2, *(D)}$ and Andronikos Paliathanasis ${ }^{3,4}$ (D) \\ 1 Faculty of Physics, Department of Astronomy-Astrophysics-Mechanics, University of Athens, \\ Panepistemiopolis, 15783 Athens, Greece; antmits@phys.uoa.gr (A.M.); mtsampa@phys.uoa.gr (M.T.) \\ 2 Departamento de Matemáticas, Universidad Católica del Norte, Avda. Angamos 0610, \\ Casilla 1280, Antofagasta, Chile \\ 3 Institute of Systems Science, Durban University of Technology, P.O. Box 1334, Durban 4000, South Africa; \\ anpaliat@phys.uoa.gr \\ 4 Instituto de Ciencias Físicas y Matemáticas, Universidad Austral de Chile, Valdivia 5090000, \\ Región de Los Ríos, Chile \\ * Correspondence: genly.leon@ucn.cl
}

Citation: Mitsopoulos, A.;

Tsamparlis, M.; Leon, G.;

Paliathanasis, A. New Conservation

Laws and Exact Cosmological

Solutions in Brans-Dicke Cosmology with an Extra Scalar Field. Symmetry 2021, 13, 1364. https://doi.org/ $10.3390 /$ sym 13081364

Academic Editor: Joan Solà Peracaula

Received: 5 July 2021

Accepted: 21 July 2021

Published: 27 July 2021

Publisher's Note: MDPI stays neutral with regard to jurisdictional claims in published maps and institutional affiliations.

Copyright: (c) 2021 by the authors. Licensee MDPI, Basel, Switzerland. This article is an open access article distributed under the terms and conditions of the Creative Commons Attribution (CC BY) license (https:/ / creativecommons.org/licenses/by/ $4.0 /)$.

\begin{abstract}
The derivation of conservation laws and invariant functions is an essential procedure for the investigation of nonlinear dynamical systems. In this study, we consider a two-field cosmological model with scalar fields defined in the Jordan frame. In particular, we consider a Brans-Dicke scalar field theory and for the second scalar field we consider a quintessence scalar field minimally coupled to gravity. For this cosmological model, we apply for the first time a new technique for the derivation of conservation laws without the application of variational symmetries. The results are applied for the derivation of new exact solutions. The stability properties of the scaling solutions are investigated and criteria for the nature of the second field according to the stability of these solutions are determined.
\end{abstract}

Keywords: first integrals; Brans-Dicke; scalar tensor; exact cosmological solutions

\section{Introduction}

The detailed analysis of recent cosmological observations indicates that the universe has been through two accelerating phases [1-4]. The current acceleration era is assumed to be driven by an unknown source known as dark energy, whose main characteristic is the negative pressure which provides an anti-gravity effect [5]. Furthermore, the early-universe acceleration era, known as inflation, is described by a scalar field, the inflaton, which is used to explain the homogeneity and isotropy of the present universe. In particular, the scalar field dominates the dynamics and explains the expansion era [6,7]. Nevertheless, the scalar field inflationary models are mainly defined on homogeneous spacetimes, or on background spaces with small inhomogeneities [8,9]. In [10] it was found that the presence of a positive cosmological constant in Bianchi cosmologies leads to expanding Bianchi spacetimes, evolving towards the de Sitter universe. That was the first result to support the cosmic "no-hair" conjecture [11,12]. This latter conjecture states that all expanding universes with a positive cosmological constant admit as asymptotic solution the de Sitter universe. The necessity of the de Sitter expansion is that it provides a rapid expansion for the size of the universe such that the latter effectively loses its memory on the initial conditions, which implies that the de Sitter expansion solves the "flatness", "horizon" and the monopole problem $[13,14]$.

In the literature scalar fields have been introduced in the gravitational theory in various ways. The simplest scalar field model is the quintessence model, which consists of a scalar field minimally coupled to gravity [15,16]. Another family of scalar fields are those which belong to the scalar-tensor theory. In this theory the scalar field is non-minimally coupled to gravity which makes it essential for the physical state of the theory. Another 
important characteristic of the scalar-tensor theories is that they are consistent with Mach's principle. The most common scalar-tensor theory is the Brans-Dicke theory [17] which is considered in this study. For other scalar-tensor theories and generalizations we refer the reader to [18-25] and references therein.

The Einstein field equations of general relativity are a set of ten nonlinear second-order partial differential equations with independent variables the spacetime coordinates and dependent variables the coefficient functions of the metric tensor. However, by assuming specific forms for the metric tensor and the existence of collineations the field equations are simplified by reduce the number of independent variables of to be separable, see for instance [26-30] and references therein. According to the cosmological principle in large scale the universe is assumed to be homogeneous, isotropic and spatially flat. This implies that the background space is described by the Friedmann-Lemaître-Robertson-Walker (FLRW) spacetime. This spacetime is characterized by the scale factor which defines the radius of the three-dimensional (3d) Euclidean space, consequently because of the existence of the spacetime isommetries, the field equations are reduced to ordinary differential equations. Since General Relativity is a second-order theory the field equations involve second-order derivatives of the scale factor. For simple cosmological fluids such as the ideal gas or the cosmological constant, the field equations can be solved explicitly [31]. However, when additional degrees of freedom are introduced, such as a scalar field, the field equations cannot be solved with the use of closed-form functions and techniques of analytic mechanics and one looks for First Integrals (FIs) which establish their (Liouville) integrability [32-35]. The standard method for the determination of FIs is Noether's theory [36]. However, there have appeared alternative geometric methods [37-42] which use the symmetries of the metric defined by the kinetic energy to determine the FIs of the dynamic equations. In the following, we shall make use of one such approach to determine the FIs (conservation laws) of the field equations.

In the present study, we consider a cosmological model in which the gravitational Action Integral is that of Brans-Dicke theory with an additional scalar field minimally coupled to gravity $[43,44]$. This two-scalar-field model belongs to the family of multi-scalar field models which have been used as unified dark energy models [45-47] or as alternative models for the description of the acceleration phases of the universe [48-51]. Indeed multifield inflationary models provide an alternative mechanism for the description of the early acceleration phase of the universe. The mechanism for the end of the inflation is much simple. Specifically, the scalar fields at the be beginning and at the end of the inflationary are not necessary the same. Thus, this can lead to different number of e-folds and affect the curvature perturbations [52,53]. The latter, in the non-adiabatic perturbations can provide detectable non-Gaussianities in the power spectrum [54]. As far as, the late-time acceleration phase, multifield cosmological models have been introduced to describe dark energy models with varying equation of state parameter which can cross the phantom divide line without the appearance of ghosts [54]. Such models can be solved the Hubbletension problem [51]. Furthermore, multi-scalar field models can attribute the additional degrees of freedom provided by the alternative theories of gravity [55-57]. The structure of the paper is as follows.

In Section 2, we define the cosmological model, and we present the gravitational field equations. In Section 3, we present some important results on the derivation of quadratic first integrals (QFIs) for a family of second-order ordinary differential equations (ODEs) with linear damping and perform a classification according to the admitted conservation laws. The results are applied to the cosmological model we consider in Section 4 where we construct the conservation laws for the gravitational field equations. Due to the nonlinearity of the field equations it is not possible to write the general solution of the field equation in closed form. However, we find some exact closed-form solutions with potential interest for the description of the cosmological history. The stability of these exact solutions is investigated in Section 5. Finally, in Section 6 we summarize our results, and we draw our conclusions. 


\section{Cosmological Model}

For the gravitational Action Integral, we consider that of Brans-Dicke scalar field theory with an additional matter source leading to the expression $[17,18]$

$$
S=\int d^{4} x \sqrt{-g}\left[\frac{1}{2} \phi R-\frac{1}{2} \frac{\omega_{B D}}{\phi} g^{\mu v} \phi_{; \mu} \phi_{; v}+L_{\psi}\left(\psi, \psi_{; \mu}\right)\right]+S_{m}
$$

where $\phi\left(x^{\kappa}\right)$ denotes the Brans-Dicke scalar field and $\omega_{B D}$ is the Brans-Dicke parameter. The action $S_{m}$ is assumed to describe an ideal gas with constant equation of state parameter and the Lagrangian function $L_{\psi}\left(\psi, \psi_{; \mu}\right)$ corresponds to the second scalar field $\psi\left(x^{\kappa}\right)$ which is assumed to be that of quintessence and minimally coupled to the Brans-Dicke scalar field. With these assumptions the Action Integral (1) takes the following form

$$
S=\int d^{4} x \sqrt{-g}\left[\frac{1}{2} \phi R-\frac{1}{2} \frac{\omega_{B D}}{\phi} g^{\mu v} \phi_{; \mu} \phi_{; v}-\frac{1}{2} g^{\mu v} \psi_{; \mu} \psi_{; v}-V(\psi)\right]+S_{m} .
$$

The gravitational field equations follow from the variation of the Action Integral (2) with respect to the metric tensor. They are

$$
G_{\mu v}=\frac{\omega_{B D}}{\phi^{2}}\left(\phi_{; \mu} \phi_{; v}-\frac{1}{2} g_{\mu \nu} g^{\kappa \lambda} \phi_{; \kappa} \phi_{; \lambda}\right)+\frac{1}{\phi}\left(\phi_{; \mu \nu}-g_{\mu \nu} g^{\kappa \lambda} \phi_{; \kappa \lambda}\right)+\frac{1}{\phi} T_{\mu v}
$$

where $G_{\mu \nu}=R_{\mu \nu}-\frac{1}{2} R g_{\mu \nu}$ is the Einstein tensor. The energy-momentum tensor $T_{\mu \nu}=$ ${ }^{\psi} T_{\mu v}+{ }^{m} T_{\mu \nu}$ where ${ }^{m} T_{\mu \nu}$ corresponds to the ideal gas and ${ }^{\psi} T_{\mu v}$ provides the contribution of the field $\psi\left(x^{k}\right)$ in the field equations.

Concerning the equations of motion for the matter source and the two scalar fields, we find ${ }^{m} T_{\mu v ; \sigma} g^{\mu \sigma}=0$, while variation with respect to the fields $\phi\left(x^{\kappa}\right)$ and $\psi\left(x^{\kappa}\right)$ provides the second-order differential equations

$$
\begin{gathered}
g^{\mu v} \phi_{; \mu v}-\frac{1}{2 \phi} g^{\mu v} \phi_{; \mu} \phi_{; v}+\frac{\phi}{2 \omega_{B D}} R=0 \\
g^{\mu v} \psi_{; \mu v}-\frac{d V}{d \psi}=0 .
\end{gathered}
$$

We assume the background space to be the Friedmann-Lemaître - Robertson-Walker (FLRW) spacetime with line element

$$
d s^{2}=-d t^{2}+a^{2}(t)\left(d x^{2}+d y^{2}+d z^{2}\right)
$$

where $a(t)$ is the scale factor of the universe and $H(t)=\frac{\dot{a}}{a}$ is the Hubble function. We note that a dot indicates derivative with respect to the cosmic time $t$.

From the line element (6) follows that the Ricci scalar is $R=6\left[\frac{\ddot{a}}{a}+\left(\frac{\dot{a}}{a}\right)^{2}\right]$. Replacing in the gravitational field Equation (3) we obtain

$$
\begin{gathered}
3\left(\frac{\dot{a}}{a}\right)^{2}=\frac{\omega_{B D}}{2}\left(\frac{\dot{\phi}}{\phi}\right)^{2}-3 \frac{\dot{a}}{a} \frac{\dot{\phi}}{\phi}+\frac{\rho_{m}+\rho_{\psi}}{\phi} \\
2 \frac{\ddot{a}}{a}+\left(\frac{\dot{a}}{a}\right)^{2}=-\frac{\omega_{B D}}{2}\left(\frac{\dot{\phi}}{\phi}\right)^{2}-2 \frac{\dot{a}}{a} \frac{\dot{\phi}}{\phi}-\frac{\ddot{\phi}}{\phi}-\frac{p_{m}+p_{\psi}}{\phi}
\end{gathered}
$$

where $\rho_{m}, p_{m}$ are the mass density and the isotropic pressure of the ideal gas; and for the quintessence field

$$
\rho_{\psi}=\frac{1}{2} \dot{\psi}^{2}+V(\psi), \quad p_{\psi}=\frac{1}{2} \dot{\psi}^{2}-V(\psi) .
$$


For the equations of motion for the scalar fields we find

$$
\ddot{\phi}+3 \frac{\dot{a}}{a} \dot{\phi}=\frac{\left(\rho_{m}-3 p_{m}\right)+\left(\rho_{\psi}-3 p_{\psi}\right)}{2 \omega_{B D}+3}
$$

and

$$
\ddot{\psi}+3 H \dot{\psi}+\frac{d V}{d \psi}=0 .
$$

Finally, for the matter source the continuity equation ${ }^{m} T_{\mu v ; \sigma} g^{\mu \sigma}=0$ reads

$$
\dot{\rho}_{m}+3 \frac{\dot{a}}{a}\left(\rho_{m}+p_{m}\right)=0 .
$$

For an ideal gas, the equation of state is $p_{m}=w_{m} \rho_{m}$, where $w_{m}$ is an arbitrary constant. Substituting in Equation (12) we find the solution

$$
\rho_{m}=\rho_{m 0} a^{-3\left(1+w_{m}\right)}
$$

where $\rho_{m 0}$ is an arbitrary constant.

The system of the ODEs that should be solved consists of the differential Equations (7), (8), (10) and (11).

\section{Quadratic First Integrals for a Class of Second-Order ODEs with Linear Damping}

Consider the second-order ODE

$$
\ddot{x}=-\omega(t) x^{n}+\Phi(t) \dot{x}
$$

where the constant $n \neq-1$. In the following we shall determine the relation between the functions $\omega(t), \Phi(t)$ for which the ODE (14) admits a quadratic first integral (QFI). The case of linear first integrals (LFIs) is also included in our study.

This problem has been considered previously in [58,59] (see Equation (28a) in [58] and Equation (17) in [59]) and has been answered partially using different methods. In [58], the author applied the Hamiltonian formalism and investigated for a canonical transformation in order to rewrite the Hamiltonian in a time-separable form. In [59], it was proposed direct approach for the derivation of FIs by multiplying the equation with an integrating factor. Moreover, in [59], it was that the two approaches are equivalent, while the the results of [59] can be seen as ageneralization of the results of [58]. Below, we extend the results of [59]. We note that a recent discussion of the same problem may be found in [60], where the authors apply more general results from the integrability of time-dependent dynamical systems of the form $\ddot{q}^{a}=-\Gamma_{b c}^{a} \dot{q}^{b} \dot{q}^{c}-\omega(t) Q^{a}(q)$.

Equation (14) is equivalent (see e.g., [61]) to the equation

$$
\frac{d^{2} x}{d \tau^{2}}=-\bar{\omega}(\tau) x^{n}, n \neq-1
$$

where the function $\bar{\omega}(\tau)$ and the new independent variable $\tau$ are defined as

$$
\tau(t)=\int e^{\int \Phi(t) d t} d t, \bar{\omega}(\tau)=\omega(t(\tau))\left(\frac{d t}{d \tau}\right)^{2} \Longleftrightarrow \omega(t)=\bar{\omega}(\tau(t)) e^{2 \int \Phi(t) d t} .
$$

We assume that equation (15) admits the general quadratic first integral

$$
I=K_{11}(\tau, x)\left(\frac{d x}{d \tau}\right)^{2}+K_{1}(\tau, x) \frac{d x}{d \tau}+K(\tau, x)
$$


where the unknown coefficients $K, K_{1}, K_{11}$ are arbitrary functions of $\tau, x$. We impose the condition

$$
\frac{d I}{d \tau}=0
$$

Replacing the second derivatives $\frac{d^{2} x}{d \tau^{2}}$, whenever they appear, using Equation (15) we find that the function $K_{11}=K_{11}(\tau)$ and the following system of equations must be satisfied

$$
\begin{aligned}
K_{1}(\tau, x) & =-\frac{d K_{11}}{d \tau} x+b_{1}(\tau) \\
K(\tau, x) & =2 \bar{\omega} K_{11} \frac{x^{n+1}}{n+1}+\frac{d^{2} K_{11}}{d \tau^{2}} \frac{x^{2}}{2}-\frac{d b_{1}}{d \tau} x+b_{2}(\tau) \\
0 & =\left(\frac{2 \frac{d \bar{\omega}}{d \tau} K_{11}}{n+1}+\frac{2 \bar{\omega} \frac{d K_{11}}{d \tau}}{n+1}+\bar{\omega} \frac{d K_{11}}{d \tau}\right) x^{n+1}-\bar{\omega} b_{1} x^{n}+\frac{d^{3} K_{11}}{d \tau^{3}} \frac{x^{2}}{2}-\frac{d^{2} b_{1}}{d \tau^{2}} x+\frac{d b_{2}}{d \tau}
\end{aligned}
$$

where $b_{1}(\tau), b_{2}(\tau)$ are arbitrary functions.

We consider the solution of the latter system (19)-(21) for various values of the power $n$.

As will be shown for the values $n=0,1,2$ there results a family of 'frequencies' $\bar{\omega}(\tau)$ parameterized with functions, whereas for the values $n \neq-1$ results a family of 'frequencies' $\bar{\omega}(\tau)$ parameterized with constants.

\subsection{Case $n=0$}

$$
\text { For } n=0 \text { the QFI (17) becomes }
$$

$I=K_{11}\left(\frac{d x}{d \tau}\right)^{2}-\frac{d K_{11}}{d \tau} x \frac{d x}{d \tau}+b_{1}(\tau) \frac{d x}{d \tau}+c_{3} x^{2}+2 \bar{\omega}(\tau) K_{11} x-\frac{d b_{1}}{d \tau} x+\int b_{1}(\tau) \bar{\omega}(\tau) d \tau$

where $K_{11}=c_{1}+c_{2} \tau+c_{3} \tau^{2}$, the parameters $c_{1}, c_{2}, c_{3}$ are arbitrary constants and the functions $b_{1}(\tau), \bar{\omega}(\tau)$ satisfy the condition

$$
\frac{d^{2} b_{1}}{d \tau^{2}}=2 \frac{d \bar{\omega}}{d \tau} K_{11}+3 \bar{\omega} \frac{d K_{11}}{d \tau} .
$$

Using the transformation (16) Equations (22) and (23) become

$$
\begin{aligned}
I & =\left[c_{1}+c_{2} \int e^{\int \Phi(t) d t} d t+c_{3}\left(\int e^{\int \Phi(t) d t} d t\right)^{2}\right] e^{-2 \int \Phi(t) d t} \dot{x}^{2} \\
& -\left[c_{2}+2 c_{3} \int e^{\int \Phi(t) d t} d t\right] e^{-\int \Phi(t) d t} x \dot{x}+b_{1}(\tau(t)) e^{-\int \Phi(t) d t} \dot{x}+c_{3} x^{2} \\
& +2 \omega(t)\left[c_{1}+c_{2} \int e^{\int \Phi(t) d t} d t+c_{3}\left(\int e^{\int \Phi(t) d t} d t\right)^{2}\right] e^{-2 \int \Phi(t) d t} x \\
& -\dot{b}_{1} e^{-\int \Phi(t) d t} x+\int b_{1}(\tau(t)) \omega(t) e^{-\int \Phi(t) d t} d t
\end{aligned}
$$

and

$$
\begin{aligned}
\ddot{b}_{1}-\Phi \dot{b}_{1} & =2 e^{-\int \Phi(t) d t}(\dot{\omega}-2 \Phi \omega)\left[c_{1}+c_{2} \int e^{\int \Phi(t) d t} d t+c_{3}\left(\int e^{\int \Phi(t) d t} d t\right)^{2}\right] \\
& +3 \omega\left[c_{2}+2 c_{3} \int e^{\int \Phi(t) d t} d t\right] .
\end{aligned}
$$




\subsection{Case $n=1$}

For $n=1$, we derive the well-known results of the one-dimensional (1d) timedependent oscillator (see e.g., $[62,63])$. Specifically, we find for the frequency $\bar{\omega}(\tau)=$ $-\frac{1}{b_{1}} \frac{d^{2} b 1}{d \tau^{2}}$ the LFI

$$
I_{1}=b_{1} \frac{d x}{d \tau}-\frac{d b_{1}}{d \tau} x
$$

and for the frequency $\bar{\omega}(\tau)=-\frac{1}{2 K_{11}} \frac{d^{2} K_{11}}{d \tau^{2}}+\frac{1}{4 K_{11}^{2}}\left(\frac{d K_{11}}{d \tau}\right)^{2}+\frac{c_{0}}{2 K_{11}^{2}}$, where $c_{0}$ is an arbitrary constant, the QFI. (For $K_{11}=\rho(\tau)^{2}$, where $\rho(\tau)$ is an arbitrary function, the QFI takes the usual form of the Lewis invariant.)

$$
I_{2}=K_{11}\left[\left(\frac{d x}{d \tau}\right)^{2}+\bar{\omega} x^{2}\right]-\frac{d K_{11}}{d \tau} x \frac{d x}{d \tau}+\frac{d^{2} K_{11}}{d \tau^{2}} \frac{x^{2}}{2} .
$$

Using the transformation (16) we deduce that the original equation

$$
\ddot{x}=-\omega(t) x+\Phi(t) \dot{x}
$$

for the frequency

$$
\omega(t)=-\rho^{-1} \ddot{\rho}+\Phi \rho^{-1} \dot{\rho}+\rho^{-4} e^{2 \int \Phi(t) d t}
$$

admits the general solution

$$
x(t)=\rho(t)(A \sin \theta+B \cos \theta)
$$

where $A, B$ are arbitrary constants, $\rho(t) \equiv \rho(\tau(t))$ and $\theta(\tau(t))=\int \rho^{-2}(t) e^{\int \Phi(t) d t} d t$.

\subsection{Case $n=2$}

For $n=2$, we derive the function $\bar{\omega}=K_{11}^{-5 / 2}$ and the QFI

$$
I=K_{11}(\tau)\left(\frac{d x}{d \tau}\right)^{2}-\frac{d K_{11}}{d \tau} x \frac{d x}{d \tau}+\left(c_{4}+c_{5} \tau\right) \frac{d x}{d \tau}+\frac{2}{3} K_{11}^{-3 / 2} x^{3}+\frac{d^{2} K_{11}}{d \tau^{2}} \frac{x^{2}}{2}-c_{5} x
$$

where $c_{4}, c_{5}$ are arbitrary constants and the function $K_{11}(\tau)$ is given by

$$
\frac{d^{3} K_{11}}{d \tau^{3}}=2\left(c_{4}+c_{5} \tau\right) K_{11}^{-5 / 2} .
$$

Using the transformation (16) the above results become

$$
\begin{gathered}
\omega(t)=K_{11}^{-5 / 2} e^{2 \int \Phi(t) d t} \\
I=K_{11} e^{-2 \int \Phi(t) d t} \dot{x}^{2}-\dot{K}_{11} e^{-2 \int \Phi(t) d t} x \dot{x}+\left[c_{4}+c_{5} \int e^{\int \Phi(t) d t} d t\right] e^{-\int \Phi(t) d t} \dot{x} \\
+\frac{2}{3} K_{11}^{-3 / 2} x^{3}+\left(\ddot{K}_{11}-\Phi \dot{K}_{11}\right) e^{-2 \int \Phi(t) d t} \frac{x^{2}}{2}-c_{5} x
\end{gathered}
$$

and

$$
\dddot{K}_{11}-3 \Phi \ddot{K}_{11}-\dot{\Phi} \dot{K}_{11}+2 \Phi^{2} \dot{K}_{11}=2\left[c_{4}+c_{5} \int e^{\int \Phi(t) d t} d t\right] e^{3 \int \Phi(t) d t} K_{11}^{-5 / 2}
$$

where now the function $K_{11}=K_{11}(\tau(t))$.

We note that for $n=2$ Equation (14), or to be more specific its equivalent (15), arises in the solution of Einstein field equations when the gravitational field is spherically symmetric and the matter source is a shear-free perfect fluid (see e.g., [64-69]). 


$$
\begin{aligned}
& \text { 3.4. Case } n \neq-1 \\
& \text { For } n \neq-1 \text { we find } b_{1}=b_{2}=0, K_{11}=c_{1}+c_{2} \tau+c_{3} \tau^{2} \text { and } \bar{\omega}(\tau)=\left(c_{1}+c_{2} \tau+\right. \\
& I=\left(c_{1}+c_{2} \tau+c_{3} \tau^{2}\right)\left(\frac{d x}{d \tau}\right)^{2}-\left(c_{2}+2 c_{3} \tau\right) x \frac{d x}{d \tau}+\frac{2}{n+1}\left(c_{1}+c_{2} \tau+c_{3} \tau^{2}\right)^{-\frac{n+1}{2}} x^{n+1}+c_{3} x^{2} \\
& \bar{\omega}(\tau)=\left(c_{1}+c_{2} \tau+c_{3} \tau^{2}\right)^{-\frac{n+3}{2}} . \\
& \omega(t)=\left[c_{1}+c_{2} \int e^{\int \Phi(t) d t} d t+c_{3}\left(\int e^{\int \Phi(t) d t} d t\right)^{2}\right]^{-\frac{n+3}{2}} e^{2 \int \Phi(t) d t} . \\
& I=\left[c_{1}+c_{2} \int e^{\int \Phi(t) d t} d t+c_{3}\left(\int e^{\int \Phi(t) d t} d t\right)^{2}\right] e^{-2 \int \Phi(t) d t} \dot{x}^{2} \\
& -\left[c_{2}+2 c_{3} \int e^{\int \Phi(t) d t} d t\right] e^{-\int \Phi(t) d t} x \dot{x} \\
& +\frac{2}{n+1}\left[c_{1}+c_{2} \int e^{\int \Phi(t) d t} d t+c_{3}\left(\int e^{\int \Phi(t) d t} d t\right)^{2}\right]^{-\frac{n+1}{2}} x^{n+1}+c_{3} x^{2} .
\end{aligned}
$$

These expressions generalize the ones given in [59]. Indeed if we introduce the notation $\omega(t) \equiv \alpha(t), \Phi(t) \equiv-\beta(t)$, then Equations (38) and (39) for $c_{3}=0$ become Equations (25) and (26) of [59].

\section{Cosmological Exact Solutions}

We can use the above results as an alternative to the Euler-Duarte-Moreira method of integrability of the anharmonic oscillator [70] in order to find exact solutions in the modified Brans-Dicke (BD) theory.

Specifically, we consider the equation of motion for the quintessence scalar field $\psi(t)$ with potential function $V(\psi)=\frac{\psi^{n+1}}{n+1}$, where $n \neq-1$. Then, Equation (11) becomes

$$
\ddot{\psi}=-\psi^{n}-3 \frac{\dot{a}}{a} \dot{\psi}
$$

which is a subcase of (14) for $\omega(t)=1$ and $\Phi(t)=-3(\ln a)$. Replacing in the transformation (16), we find that

$$
\tau(t)=\int a^{-3}(t) d t, \bar{\omega}(\tau(t))=a^{6}(t) .
$$

where Equation (40) now reads

$$
\psi^{\prime \prime}+a^{6} \psi^{n}=0
$$

where $\psi^{\prime}=\frac{d \psi(\tau)}{d \tau}$.

The latter transformation for the background space becomes

$$
d s^{2}=-a^{6}(\tau) d \tau^{2}+a^{2}(\tau)\left(d x^{2}+d y^{2}+d z^{2}\right)
$$


which means that the rest of the field equations read

$$
\begin{aligned}
6 \phi\left(\frac{a^{\prime}}{a}\right)^{2}+6 \frac{a^{\prime}}{a} \phi^{\prime}-\omega_{B D} \frac{\phi^{\prime 2}}{\phi}-\left(\psi^{\prime}\right)^{2}-\frac{2}{n+1} a^{6} \psi^{n+1} & =2 a^{6} \rho_{m} \\
4 \phi \frac{a^{\prime \prime}}{a}-10 \phi\left(\frac{a^{\prime}}{a}\right)^{2}-2 \frac{a^{\prime}}{a} \phi^{\prime}+\omega_{B D} \frac{\left(\phi^{\prime}\right)^{2}}{\phi}+2 \phi^{\prime \prime}+\left(\psi^{\prime}\right)^{2}-\frac{2}{n+1} a^{6} \psi^{n+1} & =-2 a^{6} p_{m} \\
6 \phi \frac{a^{\prime \prime}}{a}-\omega_{B D}\left[2 \phi^{\prime \prime}-\frac{\left(\phi^{\prime}\right)^{2}}{\phi}\right]-12 \phi\left(\frac{a^{\prime}}{a}\right)^{2} & =0
\end{aligned}
$$

Next, we apply the results of Section 3 for Equation (42) and we determine for several cases of the parameter $n$ the corresponding QFIs. The resulting QFIs are expressed in terms of the scale factor $a(\tau)$ and other arbitrary functions of $\tau$ (i.e., $K_{11}(\tau), b_{1}(\tau), b_{2}(\tau)$ ) which satisfy additional conditions. Solving these conditions, whenever is possible, we find a scale factor $a(\tau)$ for which Equation (42) is integrable. Replacing this scale factor in the original equation of motion (40) of the quintessence field, we end up with a new integrable second-order ODE for $\psi$ which most times can be solved using standard methods (e.g., Lie symmetries) from the symmetries of differential equations. As a final step, for the computation of exact solutions, we replace the solutions $a(t), \psi(t)$ in the remaining field Equations (7)-(10) and we determine the BD field $\phi(t)$ and the parameter $\omega_{B D}$.

\subsection{Case $n=0$}

For $n=0$ the associated QFI (22) becomes

$$
I=K_{11}\left(\psi^{\prime}\right)^{2}-K_{11}^{\prime} \psi \psi^{\prime}+b_{1}(\tau) \psi^{\prime}+c_{3} \psi^{2}+2 a^{6} K_{11} \psi-b_{1}^{\prime} \psi+\int b_{1}(\tau) a^{6} d \tau
$$

where $K_{11}=c_{1}+c_{2} \tau+c_{3} \tau^{2}$, the parameters $c_{1}, c_{2}, c_{3}$ are arbitrary constants and the functions $b_{1}(\tau), a(\tau)$ satisfy the condition

$$
b_{1}^{\prime \prime}=12 a^{5} a^{\prime} K_{11}+3 a^{6} K_{11}^{\prime} .
$$

We note that for $b_{1}=0$ we find the results of Section 4.4 below when $n=0$.

\subsection{Case $n=1$}

Using the transformation (41) equation $\psi^{\prime \prime}=-a^{6} \psi$ admits the solution

$$
\psi(\tau)=\rho(\tau)(A \sin \theta+B \cos \theta)
$$

where $\theta=\int \rho^{-2} d \tau$ and the functions $\rho(t(\tau)), a(t(\tau))$ satisfy the condition

$$
\rho^{\prime \prime}+\rho a^{6}-\rho^{-3}=0 .
$$

4.3. Case $n=2$

For $n=2$ we have $K_{11}=a^{-12 / 5}$ and the associated QFI (31) becomes

$$
I=a^{-12 / 5}\left(\psi^{\prime}\right)^{2}+\frac{12}{5} a^{-17 / 5} a^{\prime} \psi \psi^{\prime}+\left(c_{4}+c_{5} \tau\right) \psi^{\prime}+\frac{2}{3} a^{18 / 5} \psi^{3}+\frac{6}{5}\left[\frac{17}{5} a^{-22 / 5}\left(a^{\prime}\right)^{2}-a^{-17 / 5} a^{\prime \prime}\right] \psi^{2}-c_{5} \psi
$$

where $c_{4}, c_{5}$ are arbitrary constants and the function $a(t(\tau)) \equiv a(\tau)$ is given by

$$
a^{\prime \prime \prime}-\frac{51}{5} \frac{a^{\prime}}{a} a^{\prime \prime}+\frac{374}{25}\left(\frac{a^{\prime}}{a}\right)^{2} a^{\prime}+\frac{5}{6}\left(c_{4}+c_{5} \tau\right) a^{47 / 5}=0 .
$$

Substituting the given functions $\omega(t), \Phi(t)$ in Equations (33)-(35) we find equivalently that

$$
a(t)=K_{11}^{-\frac{5}{12}}
$$




$$
\begin{aligned}
I & =K_{11}^{-3 / 2} \dot{\psi}^{2}-K_{11}^{-5 / 2} \dot{K}_{11} \psi \dot{\psi}+\left(c_{4}+c_{5} \int K_{11}^{5 / 4} d t\right) K_{11}^{-5 / 4} \dot{\psi}+\frac{2}{3} K_{11}^{-3 / 2} \psi^{3} \\
& +\left[\ddot{K}_{11}-\frac{5}{4}\left(\ln K_{11}\right) \dot{K}_{11}\right] K_{11}^{-5 / 2} \frac{\psi^{2}}{2}-c_{5} \psi .
\end{aligned}
$$

where the function $K_{11}=K_{11}(t)$ is given by the differential equation

$$
\dddot{K}_{11}-\frac{15}{4}\left(\ln K_{11}\right)^{\cdot} \ddot{K}_{11}-\frac{5}{4}\left(\ln K_{11}\right)^{\cdot \cdot} \dot{K}_{11}+\frac{25}{8} \frac{\dot{K}_{11}^{3}}{K_{11}^{2}}=2\left[c_{4}+c_{5} \int K_{11}^{5 / 4} d t\right] K_{11}^{5 / 4}
$$

Equation (40) becomes $\ddot{\psi}=-\psi^{2}+\frac{5}{4}\left(\ln K_{11}\right)^{\cdot} \dot{\psi}$. We note that for $c_{4}=c_{5}=0$ we retrieve the results of Section 4.4 below for $n=2$.

In the special case with $c_{5}=0$, we find for Equation (55) the special solution $K_{11}(t)=k_{0} t^{-12}$ with constraint $c_{4} k_{0}^{1 / 4}=-192$ where $k_{0}$ is an arbitrary constant. Moreover from Equation (53) the scale factor is determined

$$
a(t)=K_{11}^{-\frac{5}{12}}=k_{0}^{-5 / 12} t^{5} .
$$

Therefore, the Klein-Gordon Equation (40) becomes

$$
\ddot{\psi}+\frac{15}{t} \dot{\psi}+\psi^{2}=0 .
$$

The latter equation can be solved by quadratures. In particular it admits the Lie symmetries

$$
\Gamma^{1}=\psi \partial_{\psi}-\frac{1}{2} t \partial_{t}, \Gamma^{2}=\left(3 \psi t^{2}-48\right) \partial_{\psi}-\frac{1}{2} t^{3} \partial_{t} .
$$

Using the vector field $\Gamma^{1}$ we find the reduced equation $\frac{1}{2} \frac{d}{d \lambda} f^{2}+2 \lambda \frac{d}{d \lambda} f+12 f+\lambda^{2}=0$ in which $f(\lambda)=t^{3} \dot{\psi}, \lambda=t^{2} \psi$. The latter equation is an Abel equation of second type. Moreover, if we assume that $\lambda$ is a constant, $\lambda=\lambda_{0}$ then we find $\psi=\lambda_{0} t^{-2}$ where by replacing in Equation (57) it follows $\lambda_{0}=24$. Therefore, we end up with the solution $\psi=\frac{24}{t^{2}}$. Let us now find the complete solution for the gravitational field equations for this particular exact solution.

Replacing these results in the rest of the field equations for dust fluid source, i.e., $p_{m}=0$ and $\rho_{m}=\rho_{0} a^{-3}$ where $\rho_{0}$ is a constant, the evolution equation for the Brans-Dicke field becomes

$$
\ddot{\phi}+\frac{15}{t} \dot{\phi}=\frac{1}{2 \omega+3}\left(\rho_{0} a^{-3}-\dot{\psi}^{2}+\frac{4}{3} \psi^{3}\right)
$$

which admits the general solution

$$
\phi(t)=-\frac{1}{2 \omega+3}\left(\frac{2016}{5} t^{-4}+\frac{\rho_{0} k_{0}^{5 / 4}}{13} t^{-13}\right)+\frac{k_{1}}{14} t^{-14}
$$

where $k_{1}$ is an arbitrary constant. Finally, by replacing in the constraint Equation (7) follows (Equation (8) is satisfied identically)

$$
\omega_{B D}=-\frac{45}{16}, k_{1}=\rho_{0}=0 .
$$

We conclude that the gravitational field equations for this model with the use of the QFI for Equation (40) admit the following exact solution

$$
\omega_{B D}=-\frac{45}{16}, a(t)=k_{0}^{-5 / 12} t^{5}, \quad \psi(t)=24 t^{-2}, \phi(t)=\frac{768}{5} t^{-4}
$$


with physical quantities

$$
\rho_{m}=p_{m}=0, \rho_{\psi}=5760 t^{-6}, p_{\psi}=-3456 t^{-6} .
$$

For the solution (58) the transformation (41) gives

$$
\tau=-\frac{k_{0}^{5 / 4}}{14} t^{-14} \Longrightarrow t=\left(-14 k_{0}^{-5 / 4}\right)^{-1 / 14} \tau^{-1 / 14} .
$$

Then the transformed field Equations (42) and (44)-(46) admit the solutions

$$
\begin{aligned}
& \omega_{B D}=-\frac{45}{16}, a=k_{0}^{-5 / 12}\left(-14 k_{0}^{-5 / 4}\right)^{-5 / 14} \tau^{-5 / 14}, \\
& \psi=24\left(-14 k_{0}^{-5 / 4}\right)^{1 / 7} \tau^{1 / 7}, \phi=\frac{768}{5}\left(-14 k_{0}^{-5 / 4}\right)^{2 / 7} \tau^{2 / 7} .
\end{aligned}
$$

\subsection{Case $n \neq-1$}

In this case, the associated QFI (36) becomes

$$
I=\left(c_{1}+c_{2} \tau+c_{3} \tau^{2}\right)\left(\psi^{\prime}\right)^{2}-\left(c_{2}+2 c_{3} \tau\right) \psi \psi^{\prime}+\frac{2}{n+1}\left(c_{1}+c_{2} \tau+c_{3} \tau^{2}\right)^{-\frac{n+1}{2}} \psi^{n+1}+c_{3} \psi^{2}
$$

and the function $a(\tau)$ is

$$
a(\tau)=\left(c_{1}+c_{2} \tau+c_{3} \tau^{2}\right)^{-\frac{n+3}{12}} .
$$

Substituting the given functions $\omega(t), \Phi(t)$ in the relation (38) we find equivalently that

$$
a^{6}(t)=\left[c_{1}+c_{2} \int a^{-3}(t) d t+c_{3}\left(\int a^{-3}(t) d t\right)^{2}\right]^{-\frac{n+3}{2}}
$$

and the associated QFI (39) becomes

$$
\begin{aligned}
I & =\left[c_{1}+c_{2} \int a^{-3}(t) d t+c_{3}\left(\int a^{-3}(t) d t\right)^{2}\right] a^{6}(t) \dot{\psi}^{2} \\
& -\left[c_{2}+2 c_{3} \int a^{-3}(t) d t\right] a^{3}(t) \psi \dot{\psi} \\
& +\frac{2}{n+1}\left[c_{1}+c_{2} \int a^{-3}(t) d t+c_{3}\left(\int a^{-3}(t) d t\right)^{2}\right]^{-\frac{n+1}{2}} \psi^{n+1}+c_{3} \psi^{2} .
\end{aligned}
$$

We consider the following special cases for which Equation (40) admits a closed-form solution for $n \neq-3,1$. In the case $n=-3$ the spacetime is that of Minkowski space. Hence, we omit the analysis.

\subsubsection{Subcase $|\tau|<<1$}

For small values of $|\tau|$ (i.e., $c_{1}=c_{3}=0$ ) the scale factor (62) is approximated as $a(\tau) \simeq \tau^{-\frac{n+3}{12}}$, therefore it follows

$$
a(t)=B_{0}\left(t-t_{0}\right)^{\frac{n+3}{3(n-1)}}
$$

where $B_{0}=\left[-\frac{c_{2}(n-1)}{4}\right]^{\frac{n+3}{3(n-1)}}$ and $t_{0}$ is an arbitrary constant.

For this asymptotic solution the equation of motion (40) for the second field $\psi$ becomes

$$
\ddot{\psi}=-\psi^{n}-\frac{n+3}{n-1} \frac{1}{t-t_{0}} \dot{\psi} .
$$

For the latter equation, the QFI (64) is

$$
I=\left[-\frac{c_{2}(n-1)}{4}\right]^{\frac{2(n+1)}{n-1}}\left(t-t_{0}\right)^{\frac{2(n+1)}{n-1}}\left(\dot{\psi}^{2}+\frac{2}{n+1} \psi^{n+1}\right)-c_{2}\left[-\frac{c_{2}(n-1)}{4}\right]^{\frac{n+3}{n-1}}\left(t-t_{0}\right)^{\frac{n+3}{n-1}} \psi \dot{\psi}
$$


This QFI for the scale factor (65) together with the results of the cases $n=0,1,2$ produce new solutions $\psi(t)$ which have not found before.

Furthermore, for the scale factor (65) the closed-form solution for the scalar field $\psi(t)$ from (66) is derived

$$
\psi(t)=\psi_{0}\left(t-t_{0}\right)^{-\frac{2}{n-1}}, \psi_{0}=\left(\frac{2}{n-1}\right)^{\frac{2}{n-1}}
$$

whereas for the BD field $\phi(t)$ it follows that $n=3, \phi(t)=\frac{\phi_{0}}{\left(t-t_{0}\right)^{2}}$ and $\omega_{B D}=-\frac{3}{2}$. However, this value for the BD parameter $\omega_{B D}$ is not physically acceptable. Hence, we do not have any close-form solution. In all discussion above we have considered $\rho_{m}=0$.

The closed-form solution found in this section is not the general solution of the field equations. That is easy to be seen since they have less free parameters from the degrees of freedom of the dynamical system. However, this form of solutions is of special interest in cosmological studies because they can describe various phases of the cosmological evolution, such as the early inflationary epoch.

\subsubsection{Subcase $|\tau| \gg 1$}

For large values of $\tau \gg 0$ (i.e., $c_{1}=c_{2}=0$ ), the scale factor (62) is approximated as $a(\tau) \simeq \tau^{-\frac{n+3}{6}}$. Therefore, in the original variable Equation (63) becomes

$$
a^{-\frac{6}{n+3}}=c_{3}^{\frac{1}{2}} \int a^{-3} d t
$$

which implies (see Equation (31) of [43])

$$
a(t)=A_{0}\left(t-t_{0}\right)^{\frac{n+3}{3(n+1)}}
$$

where $A_{0}=\left[-\frac{\sqrt{c_{3}}(n+1)}{2}\right]^{\frac{n+3}{3(n+1)}}$ and $t_{0}$ is an arbitrary constant. The scale factor $(70)$ describes a scaling solution where the effective cosmological fluid is that of an ideal gas with effective parameter for the equation of state $w_{\text {eff }}=\frac{n-1}{n+3}$. Furthermore, for $-3<n<-1,-1<$ $n<0$ the scale factor describes an accelerated universe. For $-1<n<0, w_{\text {eff }}$ is bounded as $-1<w_{\text {eff }}<-\frac{1}{3}$ while for $-3<n<-1$, $w_{\text {eff }}$ crosses the phantom divide line, i.e., $w_{\text {eff }}<-1$.

For this asymptotic solution the equation of motion (40) for the second field $\psi$ becomes

$$
\ddot{\psi}=-\psi^{n}-\frac{n+3}{n+1} \frac{1}{t-t_{0}} \dot{\psi}
$$

and the corresponding QFI (64) is written as

$$
I=c_{3}\left[\frac{(n+1)\left(t-t_{0}\right)}{2} \dot{\psi}+\psi\right]^{2}+\frac{c_{3}(n+1)}{2}\left(t-t_{0}\right)^{2} \psi^{n+1}
$$

where $t \neq t_{0}$.

However, the system admits the closed-form solution (see Equation (32) of [43])

$$
\psi(t)=\psi_{0}\left(t-t_{0}\right)^{-\frac{2}{n-1}}
$$

in which $\psi_{0}$ is given by the expression $\psi_{0}=(-2)^{\frac{3}{n-1}}\left[(n+1)(n-1)^{2}\right]^{\frac{1}{1-n}}$. Replacing in the remaining Equations (7)-(10) for the Brans-Dicke field we calculate

$$
\phi(t)=\phi_{0}\left(t-t_{0}\right)^{-\frac{4}{n-1}}
$$


in which

$$
\begin{aligned}
& \phi_{0}=\frac{(n-1)^{\frac{4}{1-n}}}{2(n+3)\left(2 \omega_{B D}+3\right)}\left[(-2)^{\frac{3(n+1)}{n-1}}(n+1)^{\frac{n+1}{1-n}}-(-2)^{\frac{6}{n-1}}(n+1)^{\frac{n-3}{n-1}}\right] \\
& \omega_{B D}=\frac{b_{1}-3 b_{2}}{1+2 b_{2}}
\end{aligned}
$$

while we have assumed that there is not any other matter source, i.e., $\rho_{m}=0$. The constants $b_{1}, b_{2}$ are given by the relations

$$
\begin{aligned}
& b_{1}=\frac{(n+3)(n-1)}{2(n+1)}\left[\frac{(n+3)(n-1)}{12(n+1)}-1\right] \\
& b_{2}=\frac{n+3}{4} \cdot \frac{2(-2)^{\frac{6}{n-1}}(n+1)^{\frac{2}{1-n}}+(-2)^{\frac{3(n+1)}{n-1}}(n+1)^{\frac{2 n}{1-n}}}{(-2)^{\frac{3(n+1)}{n-1}}(n+1)^{\frac{n+1}{1-n}}-(-2)^{\frac{6}{n-1}}(n+1)^{\frac{n-3}{n-1}}} .
\end{aligned}
$$

In the following we perform a detailed study on the stability of the latter closedform solutions.

\section{Stability of Scaling Solutions}

The analysis of the stability properties of the exact solutions provides us with important information about the evolution of the background space on the asymptotic solutions. In particular, we can infer if an exact solution is stable, which can be seen as a future attractor for the original dynamical system. On the other hand, in the cases of unstable solutions the behavior of the asymptotic solution and its dynamics give us results for the curvature and the dynamics of the metric space.

According to the methods in $[15,71,72]$ let be

$$
F(\ddot{\psi}, \dot{\psi}, \psi)=0
$$

a second-order ODE in one dimension which admits a singular power law solution

$$
\psi_{c}(t)=\psi_{0} t^{\beta}
$$

where $\psi_{0}$ is an arbitrary constant. To examine the stability of the solution $\psi_{c}$, the logarithmic time $T$ through $t=e^{T}$ is introduced, such that $t \rightarrow 0$ as $T \rightarrow-\infty$ and $t \rightarrow+\infty$ as $T \rightarrow+\infty$. We use $\psi^{\prime} \equiv \frac{d \psi}{d T}$ in the following discussion.

The following dimensionless function is introduced

$$
u(T)=\frac{\psi(T)}{\psi_{c}(T)}
$$

and the stability analysis in translated into the analysis of the stability of the equilibrium point $u=1$ of a transformed dynamical system. To construct the aforementioned system the following relations are useful:

$$
\dot{\psi}=e^{-T} \psi^{\prime}, \quad \ddot{\psi}=e^{-2 T}\left(\psi^{\prime \prime}-\psi^{\prime}\right), \quad \text { and } \quad \frac{\psi_{c}^{\prime}}{\psi_{c}}=\beta \quad \text { if } \quad \psi_{c}(t)=\psi_{0} t^{\beta} .
$$

In this section, we use a similar procedure for analyzing stability of the scaling solutions obtained in Section 4.4.

Case $|\tau| \gg 1$

For the analysis of the solution (73) of (71) we set $t_{0}=0$ by a time shift. Using (82) we have

$$
\psi^{\prime \prime}(T)=-\frac{2 \psi^{\prime}(T)}{n+1}-e^{2 T} \psi(T)^{n} .
$$


Denoting $p=-\frac{2}{n-1}$ we have

$$
\begin{aligned}
& u^{\prime \prime}(T)=\frac{p^{2} e^{-p T} \psi(T)}{\psi_{0}}+\frac{e^{-p T} \psi^{\prime \prime}(T)}{\psi_{0}}-\frac{2 p e^{-p T} \psi^{\prime}(T)}{\psi_{0}} \\
& u^{\prime}(T)=\frac{e^{-p T} \psi^{\prime}(T)}{\psi_{0}}-\frac{p e^{-p T} \psi(T)}{\psi_{0}} \\
& u(T)=\frac{e^{-p T} \psi(T)}{\psi_{0}}
\end{aligned}
$$

Hence

$$
\begin{aligned}
& \psi^{\prime \prime}(T)=\psi_{0} e^{p T}\left(p^{2} u(T)+2 p u^{\prime}(T)+u^{\prime \prime}(T)\right) \\
& \psi^{\prime}(T)=\psi_{0} e^{p T}\left(p u(T)+u^{\prime}(T)\right) \\
& \psi(T))=\psi_{0} e^{p T} u(T) .
\end{aligned}
$$

Equation (83) becomes

$$
u^{\prime \prime}(T)=\left(-\frac{2}{n+1}-2 p\right) u^{\prime}(T)+\psi_{0}^{n-1}\left(-e^{T((n-1) p+2)}\right) u(T)^{n}-\frac{p(n p+p+2) u(T)}{n+1}
$$

Substituting $p=-\frac{2}{n-1}$ and $\psi_{0}=(-2)^{\frac{3}{n-1}}\left[(n+1)(n-1)^{2}\right]^{\frac{1}{1-n}}$ it is obtained the second-order equation

$$
u^{\prime \prime}(T)=\frac{2(n+3) u^{\prime}(T)}{n^{2}-1}+\frac{8 u(T)^{n}}{(n-1)^{2}(n+1)}-\frac{8 u(T)}{(n-1)^{2}(n+1)} .
$$

Defining

$$
x=u(T), \quad y=u^{\prime}(T)
$$

we obtain the autonomous system

$$
\begin{aligned}
& x^{\prime}(T)=y(T) \\
& y^{\prime}(T)=\frac{2(n+3) y(T)}{n^{2}-1}+\frac{8 x(T)^{n}}{(n-1)^{2}(n+1)}-\frac{8 x(T)}{(n-1)^{2}(n+1)} .
\end{aligned}
$$

The scaling solution (73) is transformed to the equilibrium point $P:=(x, y)=(1,0)$. The system (93) and (94) also admits the trivial solution $O:=(x, y)=(0,0)$ as an equilibrium point and in the case that $n$ is odd, the symmetrical point $P$ given by $\bar{P}:=(x, y)=$ $(-1,0)$ is also an equilibrium point.

The linearization matrix of system (93) and (94) is

$$
J(x, y)=\left(\begin{array}{cc}
0 & 1 \\
\frac{8\left(n x^{n-1}-1\right)}{(n-1)^{2}(n+1)} & \frac{2(n+3)}{n^{2}-1}
\end{array}\right) .
$$

For $n>1, J(0,0)$ is real-valued, with eigenvalues $\left\{\frac{4}{n^{2}-1}, \frac{2}{n-1}\right\}$. Then, the origin is unstable for $n>1$.

The eigenvalues of $J(1,0)$ are $\left\{-\frac{2}{n+1},-\frac{4}{1-n}\right\}$. Therefore, $(x, y)=(1,0)$ is a sink for $-1<n<1$. It is a saddle for $n<-1$, or $n>1$.

If $n$ is odd number, say $n=2 k+1$, with $k \in \mathbb{Z}$, the eigenvalues of $J(-1,0)$ are $\left\{-\frac{1}{k+1}, \frac{2}{k}\right\}$ and when it exists, $\bar{P}$ is a saddle. 
In Figure 1, a phase-plot of system (93) and (94) for $n \in\{2,3,5,10\}$ is presented. $P$ is a saddle given $|n|>1$. When $n$ is odd, the symmetrical point $\bar{P}$ is a saddle given $|n|>1$. The origin $O$ is a source, and the orbits diverge to infinity.

In Figure 2, a phase-plot of system (93) and (94) for $n \in\{1 / 3,1 / 2,3 / 2,5 / 3\}$ is presented. When $n<1$, the power law solution $P$ is a sink, whereas in the other cases is a saddle given $|n|>1$.
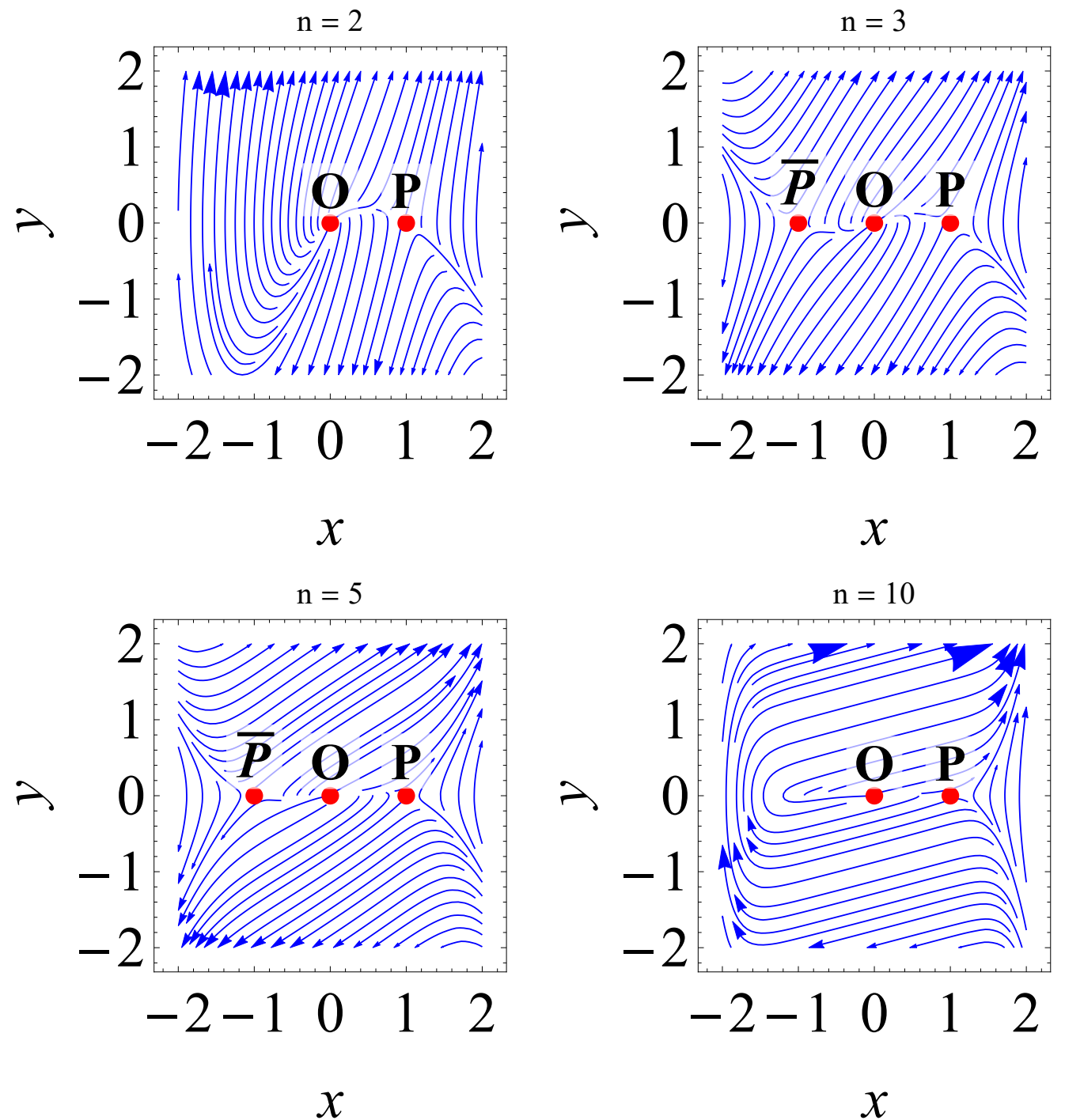

Figure 1. Phase-plot of system (93) and (94) for $n \in\{2,3,5,10\}$. $P$ is a saddle given $|n|>1$. When $n$ is odd, the symmetrical point $\bar{P}$ is a saddle given $|n|>1$. The origin $O$ is a source, and the orbits diverge to infinity. 


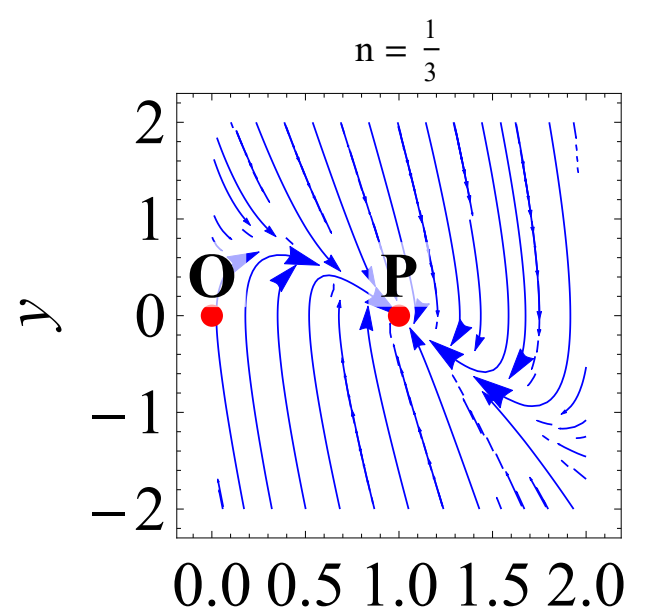

$x$

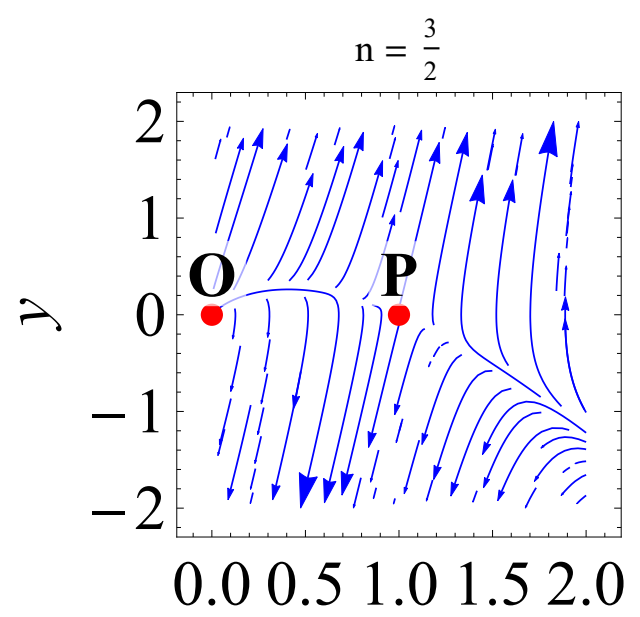

$x$

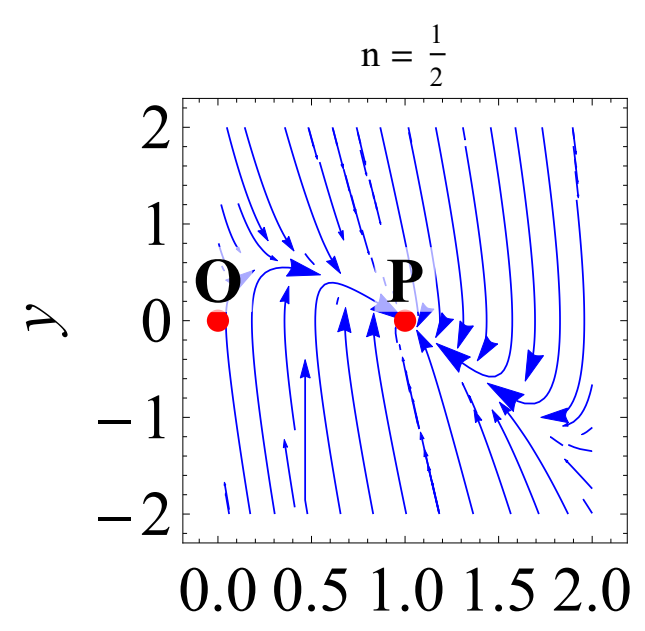

$x$

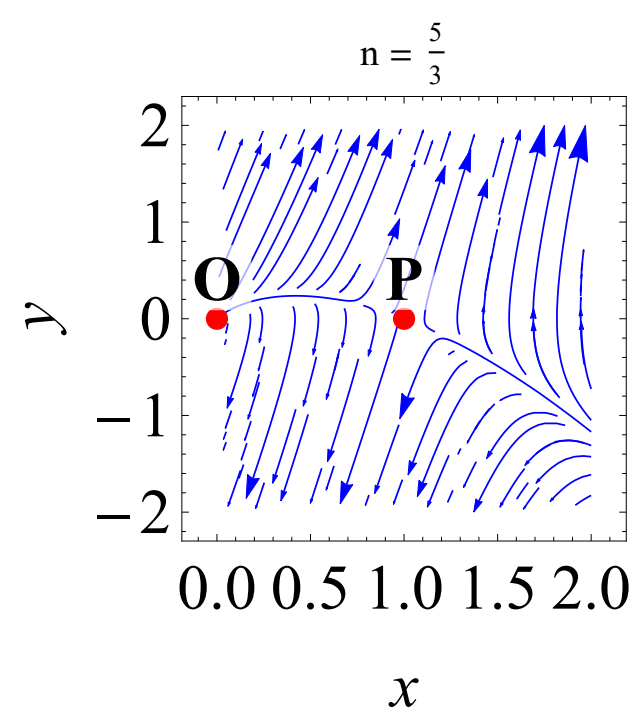

Figure 2. Phase-plot of system (93) and (94) for $n \in\{1 / 3,1 / 2,3 / 2,5 / 3\}$. When $n<1$ the power law solution $P$ is a sink, whereas in the other cases is a saddle given $|n|>1$.

\section{Conclusions}

In this work, we considered a cosmological model consisted by a Brans-Dicke field and a minimally coupled quintessence field in a spatially flat FLRW background space. For this cosmological model, the gravitational field equations consist of a Hamiltonian system of six degrees of freedom. The dynamical variables correspond to the scale factor and to the two scalar fields.

To study the integrability of the field equations, we have applied a direct method which determines the FIs of a dynamical system without the use of Noether's theorem. In this approach one assumes a generic form for the FIs, say $I$, and directly applies the condition $d I / d t=0$ using the dynamical equations. These considerations resulted in a system of partial differential equations involving the unknown coefficients defining $I$ and the dynamical quantities which characterize the dynamical system. The resulting system of equations is solved in terms of the symmetries and the Killing tensors of the kinetic metric and its solution provides the considered FIs.

For a power law scalar field potential function of the quintessence field, we found conservation laws quadratic in the first order derivatives. Using the conservation laws, we were able to find exact solutions for the field equations. In particular, we found scaling solutions for the scale factor which describe ideal gas solutions. The stability properties of 
these solutions was investigated. We were able to recover previous published results in the literature and to find new QFIs.

Using methods in $[15,71,72]$, we have studied second-order ODE in one dimension which admits a singular power law solution $\psi_{c}(t)=\psi_{0} t^{\beta}$ where $\psi_{0}$ is an arbitrary constant. To examine the stability of the solution $\psi_{c}$, the logarithmic time $T$ through $t=e^{T}$ was introduced, such that $t \rightarrow 0$ as $T \rightarrow-\infty$ and $t \rightarrow+\infty$ as $T \rightarrow+\infty$. According to our analysis, the scaling solution (73) is transformed to the equilibrium point $P:=(x, y)=$ $(1,0)$, which is a sink for $-1<n<1$ or a saddle for $n<-1$, or $n>1$. The dynamical system also admits the trivial solution $O:=(x, y)=(0,0)$ as an equilibrium point and in the case that $n$ is an odd, the symmetrical point $P$ given by $\bar{P}:=(x, y)=(-1,0)$ is also an equilibrium point. The origin is unstable for $n>1$. If $n$ is an odd number, the point $\bar{P}$ exists and is a saddle.

Until now, most of this kind of studies, for the investigation of conservation laws, have been done mainly with the application of variational symmetries. Our approach is more general and does not required the existence of a point-like Lagrangian, i.e., of a minisuperspace description. Therefore, this generic approach can be applied in other gravitational models without minisuperspace such are the Class B Bianchi spacetimes.

Author Contributions: A.M. and M.T. established the research problem and proved the results presented in Sections 3 and 4. G.L. worked on the stability analysis of Section 5. Finally, A.P. organized the plan of the paper and discussed the physical interpretation of the results. All authors have read and agreed to the published version of the manuscript.

Funding: The research of AP and GL was funded by Agencia Nacional de Investigación y DesarrolloANID through the program FONDECYT Iniciación grant no. 11180126. Additionally, GL was funded by Vicerrectoría de Investigación y Desarrollo Tecnológico at Universidad Católica del Norte. This work is based on the research supported in part by the National Research Foundation of South Africa (Grant Numbers 131604).

Institutional Review Board Statement: Not applicable.

Informed Consent Statement: Not applicable.

Data Availability Statement: Not applicable.

Conflicts of Interest: The authors declare no conflict of interest.

\section{References}

1. Riess, A.G.; Filippenko, A.V.; Challis, P.; Clocchiatti, A.; Diercks, A.; Garnavich, P.M.; Gilliland, R.L.; Hogan, C.J.; Jha, S.; Kirshner, R.P.; et al. Observational Evidence from Supernovae for an Accelerating Universe and a Cosmological Constant. Astron. J. 1998, 116, 1009-1038. [CrossRef]

2. Perlmutter, S.; Aldering, G.; Deustua, S.; Fabbro, S.; Goldhaber, G.; Groom, D.E.; Kim, A.G.; Kim, M.Y.; Knop, R.A.; Nugent, P.; et al. Cosmology from Type Ia supernovae. Bull. Am. Astron. Soc. 1997, 29, 1351.

3. Riess, A.G.; Strolger, L.; Casertano, S.; Ferguson, H.C.; Mobasher, B.; Gold, B.; Challis, P.J.; Filippenko, A.V.; Jha, S.; Li, W.; et al. NewHubble Space TelescopeDiscoveries of Type Ia Supernovae at $\mathrm{z}>=1$ : Narrowing Constraints on the Early Behavior of Dark Energy. Astrophys. J. 2007, 659, 98-121. [CrossRef]

4. Suzuki, N.; Rubin, D.; Lidman, C.; Aldering, G.S.; Amanullah, R.; Barbary, K.; Barrientos, L.F.; Botyanszki, J.; Brodwin, M.; Connolly, N.; et al. Thehubble space telescopecluster supernova survey. v. improving the dark-energy constraints abovez $>1$ and building an early-type-hosted supernova sample. Astrophys. J. 2012, 746, 85. [CrossRef]

5. di Valentino, E.; Mena, O.; Pan, S.; Visinelli, L.; Yang, W.; Melchiorri, A.; Mota, D.F.; Riess, A.G.; Silk, J. In the realm of the Hubble tension-A review of solutions. Class. Quantum Gravity 2021, 2103. [CrossRef]

6. Starobinsky, A. A new type of isotropic cosmological models without singularity. Phys. Lett. B 1980, 91, 99-102. [CrossRef]

7. Guth, A.H. Inflationary universe: A possible solution to the horizon and flatness problems. Phys. Rev. D 1981, $23,347-356$. [CrossRef]

8. Müller, V.; Schmidt, H.-J.; Starobinsky, A. The stability of the de Sitter space-time in fourth order gravity. Phys. Lett. B 1988, 202, 198-200. [CrossRef]

9. Kofman, L.; Linde, A.; Starobinsky, A. Inflationary universe generated by the combined action of a scalar field and gravitational vacuum polarization. Phys. Lett. B 1985, 157, 361-367. [CrossRef]

10. Wald, R.M. Asymptotic behavior of homogeneous cosmological models in the presence of a positive cosmological constant. Phys. Rev. D 1983, 28, 2118-2120. [CrossRef] 
11. Gibbons, G.W.; Hawking, S.W. Cosmological event horizons, thermodynamics, and particle creation. Phys. Rev. D 1977, 15, 2738-2751. [CrossRef]

12. Hawking, S.; Moss, I. Supercooled phase transitions in the very early universe. Phys. Lett. B 1982, 110, 35-38. [CrossRef]

13. Sato, K. First-order phase transition of a vacuum and the expansion of the Universe. Mon. Not. R. Astron. Soc. 1981, 195, 467-479. [CrossRef]

14. Barrow, J.D.; Ottewill, A. The stability of general relativistic cosmological theory. J. Phys. A Math. Gen. 1983, 16, 2757-2776. [CrossRef]

15. Ratra, B.; Peebles, P.J.E. Cosmological consequences of a rolling homogeneous scalar field. Phys. Rev. D 1988, 37, 3406-3427. [CrossRef]

16. Barrow, J.D.; Saich, P. Scalar-field cosmologies. Class. Quantum Gravity 1993, 10, 279-283. [CrossRef]

17. Brans, C.H.; Dicke, R.H. Mach's Principle and a Relativistic Theory of Gravitation. Phys. Rev. 1961, 124, 925-935. [CrossRef]

18. Faraoni, V. Cosmology in Scalar-Tensor Gravity, Fundamental Theories of Physics; Kluwer Academic Press: Dordrecht, The Netherlands, 2004; Volume 139.

19. Horndeski, G.W. Second-order scalar-tensor field equations in a four-dimensional space. Int. J. Theor. Phys. 1974, 10, 363-384. [CrossRef]

20. O'Hanlon, J. Intermediate-Range Gravity: A Generally Covariant Model. Phys. Rev. Lett. 1972, 29, 137-138. doi:10.1103/physrevlett.29.137. [CrossRef]

21. Nicolis, A.; Rattazzi, R.; Trincherini, E. Galileon as a local modification of gravity. Phys. Rev. D 2009, 79, 064036. [CrossRef]

22. Deffayet, C.; Esposito-Farese, G.; Vikman, A. Covariant Galileon. Phys. Rev. D 2009, 79. [CrossRef]

23. Belinchón, J.A.; Harko, T.; Mak, M.K. Exact scalar-tensor cosmological models. Int. J. Mod. Phys. D 2017, 26, 1750073. [CrossRef]

24. Fomin, I.V.; Chervon, S.V. Exact and slow-roll solutions for exponential power-law inflation connected with $\mathrm{f}(\mathrm{R})$ gravity and observational constraints. arXiv 2006, arXiv:2006.16074.

25. Fomin, I.V.; Chervon, S.; Maharaj, S.D. A new look at the Schrödinger equation in exact scalar field cosmology. Int. J. Geom. Methods Mod. Phys. 2019, 16, 1950022. [CrossRef]

26. Saridakis, E.; Tsamparlis, M. Symmetry inheritance of conformal Killing vectors. J. Math. Phys. 1991, 32, 1541-1551. [CrossRef]

27. Shapovalov, V.N. Stäckel spaces. Sib. Math. J. 1979, 20, 790. [CrossRef]

28. Obukhov, V. Separation of variables in Hamilton-Jacobi and Klein-Gordon-Fock equations for a charged test particle in the Stäckel spaces of type. Int. J. Geom. Methods Mod. Phys. 2021, 18, 2150033. [CrossRef]

29. Osetrin, E.; Osetrin, K. Pure radiation in space-time models that admit integration of the eikonal equation by the separation of variables method. J. Math. Phys. 2017, 58, 112504. [CrossRef]

30. Tsamparlis, M.; Paliathanasis, A. The generic model of general relativity. Arab. J. Math. 2019, 8, 201-254. [CrossRef]

31. Amendola, L.; Tsujikawa, S. Dark Energy: Theory and Observations; Cambrdige University Press: Cambridge, UK, 2010.

32. Demiański, M.; De Ritis, R.; Marmo, G.; Platania, G.; Rubano, C.; Scudellaro, P.; Stornaiolo, C. Scalar field, nonminimal coupling, and cosmology. Phys. Rev. D 1991, 44, 3136-3146. [CrossRef] [PubMed]

33. Dimakis, N.; Giacomini, A.; Paliathanasis, A. Integrability from point symmetries in a family of cosmological Horndeski Lagrangians. Eur. Phys. J. C 2017, 77, 458. [CrossRef]

34. Dimakis, N.; Terzis, P.A.; Christodoulakis, T. Integrability of the mixmaster model. Phys. Rev. D 2019, 99, 023536. [CrossRef]

35. Papagiannopoulos, G.; Barrow, J.D.; Basilakos, S.; Giacomini, A.; Paliathanasis, A. Dynamical symmetries in Brans-Dicke cosmology. Phys. Rev. D 2017, 95, 024021. [CrossRef]

36. Tsamparlis, M.; Paliathanasis, A. Symmetries of Differential Equations in Cosmology. Symmetry 2018, 10, 233. [CrossRef]

37. Katzin, G.H. Related integral theorem. II. A method for obtaining quadratic constants of the motion for conservative dynamical systems admitting symmetries. J. Math. Phys. 1973, 14, 1213-1217. [CrossRef]

38. Katzin, G.H.; Levine, J. Geodesic first integrals with explicit path-parameter dependence in Riemannian space-times. J. Math. Phys. 1981, 22, 1878-1891. [CrossRef]

39. Katzin, G.H.; Levine, J. Time-dependent quadratic constants of motion, symmetries, and orbit equations for classical particle dynamical systems with time-dependent Kepler potentials. J. Math. Phys. 1982, 23, 552-563. [CrossRef]

40. Horwood, J.T. Higher order first integrals in classical mechanics. J. Math. Phys. 2007, 48, 102902. [CrossRef]

41. Tsamparlis, M.; Mitsopoulos, A. Quadratic first integrals of autonomous conservative dynamical systems. J. Math. Phys. 2020, 61, 072703. [CrossRef]

42. Tsamparlis, M.; Mitsopoulos, A. First integrals of holonomic systems without Noether symmetries. J. Math. Phys. 2020, 61, 122701. [CrossRef]

43. Mukherjee, P.; Chakrabarti, S. Exact solutions and accelerating universe in modified Brans-Dicke theories. Eur. Phys. J. C 2019, 79, 681. [CrossRef]

44. Giacomini, A.; Leon, G.; Paliathanasis, A.; Pan, S. Cosmological evolution of two-scalar fields cosmology in the Jordan frame. Eur. Phys. J. C 2020, 80, 1-31. [CrossRef]

45. Cid, A.; Leon, G.; Leyva, Y. Intermediate accelerated solutions as generic late-time attractors in a modified Jordan-Brans-Dicke theory. J. Cosmol. Astropart. Phys. 2016, 2016, 27. [CrossRef]

46. Jamil, M.; Momeni, D.; Myrzakulov, R. Noether symmetry of $\mathrm{F}(\mathrm{T})$ cosmology with quintessence and phantom scalar fields. Eur. Phys. J. C 2012, 72, 2137. [CrossRef] 
47. Paliathanasis, A. Dynamics of chiral cosmology. Class. Quantum Gravity 2020, 37, 195014. [CrossRef]

48. Dimakis, N.; Paliathanasis, A. Crossing the phantom divide line as an effect of quantum transitions. Class. Quantum Gravity 2021, 38, 075016. [CrossRef]

49. Brown, A.R. Hyperbolic Inflation. Phys. Rev. Lett. 2018, 121, 251601. [CrossRef] [PubMed]

50. Coley, A.A.; Hoogen, R.V.D. Dynamics of multi-scalar-field cosmological models and assisted inflation. Phys. Rev. D 2000, 62, 023517. [CrossRef]

51. Cai, Y.-F.; Saridakis, E.; Setare, M.R.; Xia, J.-Q. Quintom cosmology: Theoretical implications and observations. Phys. Rep. 2010, 493, 1-60. [CrossRef]

52. Choi, K.-Y.; Kim, S.A.; Kyae, B. Primordial curvature perturbation during and at the end of multi-field inflation. Nucl. Phys. B 2012, 861, 271-289. [CrossRef]

53. Lyth, D.H. Generating the curvature perturbation at the end of inflation. JCAP 2005, 11, 6. [CrossRef]

54. Langlois, D.; Renaux-Peterl, S. Perturbations in generalized multi-field inflation. JCAP 2008, 804, 17. [CrossRef]

55. Nojiri, S.; Odintsov, S.; Oikonomou, V. Ghost-free F (R) gravity with Lagrange multiplier constraint. Phys. Lett. B 2017, 775, 44-49. [CrossRef]

56. Capozziello, S.; Matsumoto, J.; Nojiri, S.; Odintsov, S.D. Dark energy from modified gravity with Lagrange multipliers. Phys. Lett. B 2010, 693, 198-208. [CrossRef]

57. Chervon, S.V.; Fomin, I.V.; Pozdeeva, E.O.; Sami, M.; Vernov, S.Y. Superpotential method for chiral cosmological models connected with modified gravity. Phys. Rev. D 2019, 100, 063522. [CrossRef]

58. da Silva, M.R.M.C. A transformation approach for finding first integrals of motion of dynamical systems. Int. J. Non Linear Mech. 1974, 9, 241. [CrossRef]

59. Sarlet, W.; Bahar, L. A direct construction of first integrals for certain non-linear dynamical systems. Int. J. Non-Linear Mech. 1980, 15, 133-146. [CrossRef]

60. Mitsopoulos, A.; Tsamparlis, M. Quadratic first integrals of time-dependent dynamical systems of the form $\ddot{q}^{a}=-\Gamma_{b c}^{a} \dot{q}^{b} \dot{q}^{c}-$ $\omega(t) Q^{a}(q)$. Mathematics 2021, 9, 1503. [CrossRef]

61. Karpathopoulos, L.; Paliathanasis, A.; Tsamparlis, M. Lie and Noether point symmetries for a class of nonautonomous dynamical systems. J. Math. Phys. 2017, 58, 082901. [CrossRef]

62. Katzin, G.H.; Levine, J. Dynamical symmetries and constants of the motion for classical particle systems. J. Math. Phys. 1974, 15, 1460-1470. [CrossRef]

63. Prince, G.E.; Eliezer, C.J. Symmetries of the time-dependent N-dimensional oscillator. J. Phys. A Math. Gen. 1980, 13, 815-823. [CrossRef]

64. Stephani, H.; Kramer, D.; Maccallum, M.; Hoenselaers, C.; Herlt, E. Exact Solutions to Einstein's Field Equations, 2nd ed.; Cambridge University Press: New York, NY, USA, 2009.

65. Stephani, H. A new interior solution of Einstein's field equations for a spherically symmetric perfect fluid in shear-free motion. J. Phys. A Math. Gen. 1983, 16, 3529-3532. [CrossRef]

66. Srivastana, D.C. Exact solutions for shear-free motion of spherically symmetric perfect fluid distributions in general relativity. Class. Quant. Grav. 1987, 4, 1093. [CrossRef]

67. Leach, P.G.L.; Maharaj, S.D. A first integral for a class of time-dependent anharmonic oscillators with multiple anharmonicities. J. Math. Phys. 1992, 33, 2023-2030. [CrossRef]

68. Leach, P.G.L.; Maartens, R.; Maharaj, S.D. Self-similar solutions of the generalized Emden-Fowler equation. Int. J. Non-Linear Mech. 1992, 27, 575. [CrossRef]

69. Maharaj, S.D.; Leach, P.G.L.; Maartens, R. Expanding spherically symmetric models without shear. Gen. Relativ. Gravit. 1996, 28, 35-50. [CrossRef]

70. Duarte, L.G.S.; Moreira, I.C.; Euler, N.; Steeb, W.-H. Invertible point transformations, Lie symmetries and the Painlevé test for the equation $\mathrm{d}^{2} x / \mathrm{d} t^{2}+f_{1}(\mathrm{t}) \mathrm{d} x / \mathrm{d} t+f_{2}(t) x+f_{3}(t) x^{n}=0$. Phys. Scr. 1991, 43, 449-451. [CrossRef]

71. Liddle, A.; Scherrer, R.J. Classification of scalar field potentials with cosmological scaling solutions. Phys. Rev. D 1998, 59, 023509. [CrossRef]

72. Uzan, J.P. Effects of a scalar fifth force on the dynamics of a charged particle as a new experimental design to test chameleon theories. Phys. Rev. D 1999, 59, 123510. [CrossRef] 